[Technical Paper】

\title{
Structure and Properties of Graphene Oxide-Chitosan Composite Membrane Prepared by the Plasma Acid Solvent
}

\author{
Lei Zhang*1,2, Ying Wang*1,\#, and Xing Yang*1 \\ ${ }^{* 1}$ School of Textile and Material Engineering, Dalian Polytechnic University, Dalian 116034, China \\ ${ }^{*}$ Dalian Ruiguang Nonwoven Group Co. Ltd., Dalian 116100, China
}

\begin{abstract}
In order to obtain a green solvent for chitosan, plasma acid was prepared by dielectric barrier discharge of water. The results showed that the maximum saturated solubility of chitosan in the plasma acid at $\mathrm{pH}$ of 1.25 was $1 \%$. Then $1 \%$ chitosan membrane, $0.5 \%$ graphene oxide (GO) and $1 \%$ chitosan composite membrane were prepared using plasma acid as solvent. The structure and properties of GO/chitosan composite membrane were investigated carefully by thermal-gravimetric, scanning electron microscope, X-ray diffraction, Fourier transform infrared spectroscopy and mechanical property analysis. After adding GO into the chitosan solution, the GO was dispersed uniformly in the matrix. There was a strong interaction between GO and chitosan matrix, which prevented the aggregation of graphene effectively. The surface of the composite membrane became smooth. The thermal stability of $\mathrm{GO} /$ chitosan composite membrane was increased. It had good mechanical properties. In a word, plasma acid could be used as a green solvent, the performance of chitosan membrane was improved after adding GO. It has the potential application in the field of biomedicine and textiles.

(Received 5 February, 2017; Accepted 12 July, 2017)
\end{abstract}

\section{Introduction}

Chitosan, (1, 4) - 2 - amino - 2 - $\beta$ - deoxy - D - glucose, is the most abundant natural polymer except cellulose. It is the deacetylated product of chitin, the only basic polysaccharide in nature. It has good biocompatibility, biodegradation, adsorption, antibacterial ability and film-forming properties. So the application areas of chitosan [1-3] are expanding because of its excellent properties. The demand for chitosan is continuously increasing.

However, due to the hydrogen bonds between molecules and high crystallinity of chitosan, it is not dissolve in common organic solvents, which greatly limits the application of chitosan. Chitosan is only dissolving in some acidic aqueous solutions, including sulphuric acid, acetic acid, formic acid, and hydrofluoric acid. Knowing the disadvantages of environmentally-unfriendly, separation and recovery of these solutions, it is highly desirable to seek a green solvent of chitosan.

Dielectric barrier discharge (DBD) of water produces a large number of oxygen based species.
The reaction of these plasma species with water forms a hydrated proton $\left(\mathrm{H}_{3} \mathrm{O}^{+}\right)$. The obtained $\mathrm{H}_{3} \mathrm{O}^{+}$ has acidity. The concept of plasma acid was initially presented by Liu and Zou for the hydrolysis of starch catalyzed by DBD plasma [4]. Plasma acid has a great advantage over some frequently used inorganic acids, such as sulphuric acid, hydrochloric acid, phosphoric acid, etc. It can be obtained and separated more easily than them, because of coming from water.

Graphene oxide (GO) is the precursor of graphene prepared by chemical method. GO is one of the important graphene derivatives. It has many oxygencontaining functional groups, such as hydroxyl, carboxyl and epoxy group. It is a kind of good active material [5]. For these reactive groups amenable for chemical modifications, GO is tailored for a range of biomedical and other applications [6-8]. For the GO/ chitosan composite material, the molecular structure of chitosan contains amino groups, which can react with carboxyl groups in GO to form -NHCO- bond [9], chitosan and GO are closely connected together to form a composite material, showed some special properties. 
The mechanical properties and heat resistance of pure chitosan membrane have a limitation, which greatly limits the scope of application of this material. Therefore, in this work plasma acid was prepared and used as the solvent of chitosan. Then GO was added into the chitosan solution. A GO/chitosan composite solution was made and then $\mathrm{GO} /$ chitosan composite membrane was made. The groups of GO can improve the interfacial interaction between GO and matrix materials, and improve the properties of nanocomposites [10]. Then the mechanical properties, thermal stability and crystallization of composite membrane were analyzed through mechanical testing, thermal-gravimetric (TG), scanning electron microscope (SEM), X-ray diffraction (XRD) and Fourier transform infrared spectroscopy (FTIR).

\section{Experimental}

\subsection{Materials}

Chitosan (DAC $\geq 85 \%$ ) was purchased from Jinan Haidebei Marine Biological Engineering Co., Ltd (China). GO (purity $\geq 99 \%$ ) was purchased from Tangshan Jianhua Technology Development Co., Ltd (China). $\mathrm{NaOH}$ was purchased from Tianjin Kermel Chemical Reagent Co., Ltd (China).

\subsection{Preparation of plasma acid}

The plasma treatment equipment, CTP-2000 K low temperature plasma power, made by Nanjing Suman Electronics Co., Ltd (China). It was used to produce plasma acid. Before the plasma discharging, $5 \mathrm{ml}$ of distilled water was placed on the surface of the quartz electrode. Then, it was treated by plasma at discharge power of $125 \mathrm{~W}$ and discharge gap of $8 \mathrm{~mm}$. After plasma treatment, the plasma acid was obtained and used to prepare chitosan/plasma acid solution immediately.

\subsection{Effect of discharge time on the $\mathrm{pH}$ of plasma acid}

$5 \mathrm{ml}$ of distilled water was put into the plasma reaction vessel. Under the experimental conditions of 2.2, plasma acid with acidic characteristics was prepared. Then the plasma acid was sucked out by pipette and diluted by adding a proper amount of distilled water and diluted for 10 times. The $\mathrm{pH}$ value was measured by $\mathrm{pH}$ meter PHSJ-4 A, made by Shanghai Electrical Apparatus Science Instrument Co., Ltd (China). Then, according to the measured data and dilution relationships, the curve between discharge time and $\mathrm{pH}$ value was drawn, in which the horizontal coordinate was the discharge time (s) and the ordinate was the $\mathrm{pH}$ of plasma acid. The experimental scheme was shown in Table 1.

The $\mathrm{pH}$ value of plasma acid was tested every $2 \mathrm{~h}$, and a timeliness curve of plasma acid was obtained.

\subsection{Preparation of chitosan/plasma acid solution}

The $1 \%$ and $1.5 \%$ chitosan powders were added into $5 \mathrm{ml}$ plasma acid respectively. The solutions were sealed and placed on the magnetic stirrer to prepare chitosan/plasma acid solution at the temperature of $25^{\circ} \mathrm{C}$. The stirring time was about $3-4 \mathrm{~h}$ on the magnetic stirrer, RT 10 power mixer, made by IKA (Germany). The conductivity and surface tension of chitosan/plasma solution were determined by DDS307 conductivity meter, made by Shanghai Electrical Apparatus Science Instrument Limited Co., Ltd (China), and the K100C automatic surface tension meter, made by KRUSS GmbH (Germany).

\subsection{Preparation of $\mathrm{GO} /$ chitosan composite solution}

The $0.5 \% \mathrm{GO}$ was added into the chitosan/plasma acid solution to prepare $\mathrm{GO} /$ chitosan composite solution at the temperature of $25^{\circ} \mathrm{C}$. The stirring time was $4 \mathrm{~h}$.

2.6 Preparation of GO/chitosan composite membrane Chitosan/plasma acid solution and GO/chitosan

Table 1 Plasma treatment under normal temperature and pressure

\begin{tabular}{cccc}
\hline Number & Power $(\mathrm{W})$ & Discharge gap $(\mathrm{mm})$ & Time $(\mathrm{s})$ \\
\hline 1 & 125 & 8 & 10 \\
2 & 125 & 8 & 30 \\
3 & 125 & 8 & 60 \\
4 & 125 & 8 & 180 \\
5 & 125 & 8 & 300 \\
6 & 125 & 8 & 420 \\
7 & 125 & 8 & 600 \\
8 & 125 & 8 & 900 \\
\hline
\end{tabular}


composite solution were de-bubbled in the vacuum oven, DZF-6020 vacuum drying oven, made by Shanghai Yiheng Scientific Instrument Co., Ltd (China). Then the solution was put on a clean ceramic plate using the method of tape casting to prepare the membrane. A glass rod was used to control the thickness of the liquid membrane. Finally, place the membrane in a vacuum drying oven. The drying time was $15 \mathrm{~h}$ at room temperature.

After drying, $1 \% \mathrm{NaOH}$ solution was added to neutralize the plasma acid on the surface of the membrane. Then it was washed and dried for 15-20 minutes at room temperature for further measurements.

\subsection{TEM analysis}

A small amount of GO was dissolved in ethanol solution and dispersed by ultrasonic wave. Eventually it was fully dissolved and dispersed in solvent ethanol. The microstructure of GO was analyzed by JEM-2100 (UHR) transmission electron microscopy, made by Japan Electronic Co., Ltd. Technical parameters and indicators were as follows: point resolution was $0.19 \mathrm{~nm}$, line resolution was $0.14 \mathrm{~nm}$, acceleration voltage was $200 \mathrm{kV}$ and magnification was 50 $1,500,000$.

\subsection{XPS analysis}

The oxygen content of GO was tested by XPS instrument, made by Thermo ESCALAB $250 \mathrm{Xi}$ (America). Test conditions are as follows, monochrome AI Kaa (hvn=1486.6 eV), Power was $150 \mathrm{~W}$, beam spot was $500 \mu \mathrm{m}$, analyzer fixed penetration energy was $30 \mathrm{eV}$.

\subsection{FTIR analysis}

The FTIR analyzer was made by American Perkin Elmer Co., Ltd. The chitosan membranes were tested in the range from $450-4000 \mathrm{~cm}^{-1}$.

\subsection{XRD analysis}

The D/max-3B type X-ray crystal diffraction, made by Japan Science Co., Ltd, was used to analyze samples under radiation of $\mathrm{Cu}$ with the tube, tube voltage of $40.0 \mathrm{kV}$ and tube current of $30.0 \mathrm{~mA}$. The scanning range was $5^{\circ}-55^{\circ}$, and the scanning speed was $5 \%$ min.

\subsection{SEM analysis}

SEM, JSM-5600 LV scanning electron microscope, made by Japan Electronic Co., Ltd, was used to observe the surface morphology of the membranes. The images were collected at different magnification.

\subsection{TG analysis}

The chitosan membranes were cut into powders and TG was used to test thermal stability of the membranes. The HCT microcomputer differential thermal balance was made by Beijing Permanent Scientific Instrument Factory (China). Conditions were as follows: the nitrogen atmosphere, start quality of about $5 \mathrm{mg}$, the initial temperature of $35^{\circ} \mathrm{C}$, the termination temperature of $400^{\circ} \mathrm{C}$ and heating rate of $10^{\circ} \mathrm{C} / \mathrm{min}$.

\subsection{Mechanical property analysis}

The digital fabric thickness instrument YG141LA, made by Laizhou Electronic Instrument Co., Ltd (China), was used to determine thickness of two kinds of membrane. Its accuracy was $0.001 \mathrm{~mm}$. Each membrane was tested three times as follows and average value was obtained.

The membrane was cut to $5 \mathrm{~cm} \times 1 \mathrm{~cm}$. The LLY-06 E electronic single fibre tensile strength tester, made by Laizhou Electronic Instrument Co., Ltd (China), was used to test the tensile strength. Each membrane was tested three times and the average value was obtained, according to the following equation (1) to calculate the tensile strength of the membrane:

$$
\sigma=\mathrm{F} /(100 \times \mathrm{H} \times \mathrm{d})
$$

where $\sigma$ was the tensile breaking strength $(\mathrm{MPa})$, $\mathrm{F}$ was the fracture force of specimen $(\mathrm{cN}), \mathrm{H}$ was the width of specimen $(\mathrm{mm})$, and $\mathrm{D}$ was the thickness of specimen (mm).

\section{Results and discussion}

\subsection{The solution of chitosan in the plasma acid}

Previous reports [11-12] have shown that a large amount of oxygen species, including $\mathrm{O}$ atoms, $\mathrm{OH}$. radicals and $\mathrm{HO}_{2}$. radicals, were produced during the reaction of producing plasma species.

The following equation(2) illustrates the interaction of $\mathrm{HO}_{2}$-radicals with water, which is in agreement with the literature [13] showing that the $\mathrm{HO}_{2} \cdot$ radicals are a better proton donor but a weaker proton accepter in water. It is clear that this reaction resulted in the formation of a hydrated proton $\mathrm{H}_{3} \mathrm{O}^{+}$. It is the plasma product in the liquid water that originally presented a strongly acidic feature. The $\mathrm{pH}$ of plasma acid varies with the discharge conditions.

$$
\mathrm{HO}_{2} \cdot+\mathrm{H}_{2} \mathrm{O} \longleftrightarrow\left[\mathrm{O}_{2}-\mathrm{H}-\mathrm{OH}_{2}\right] \longleftrightarrow \mathrm{O}_{2-}+\mathrm{H}_{3} \mathrm{O}^{+}
$$


The above analysis showed that the plasma acid has a potential ability to dissolve the chitosan. Then the chitosan was dissolved in the plasma acid.

\subsection{Effect of discharge time on the $\mathrm{pH}$ of plasma acid}

Figure 1 was the variation curve of plasma acid with discharge time. As can be seen from the figure, when the distilled water was treated of $10 \mathrm{~s}$, its $\mathrm{pH}$ value was 6.53 . The water became neutral to acidic. In the initial stage of discharge (10-60 s), the change of $\mathrm{pH}$ value of plasma was largely reduced from 6.53 to 3.38, which showed that the discharge time has a great influence on the $\mathrm{pH}$ value of plasma acid in the initial stage of discharge. With the prolonging of discharge time (60-420 s), the $\mathrm{pH}$ value of plasma acid continued to decrease acidity, reduced from 3.38 to 1.52. During this period, the $\mathrm{H}_{3} \mathrm{O}^{+}$of plasma acid continued to increase, but the change was smaller than the initial stage of $10-60 \mathrm{~s}$. This result showed that the influence of discharge time on the acidity of the plasma became smaller. In the later stage of discharge $(600-900 \mathrm{~s})$, the $\mathrm{pH}$ value of plasma acid changed from 1.25 to 1.17. At this stage, the change in $\mathrm{pH}$ value of the plasma acid was the smallest, which indicated that the $\mathrm{pH}$ value of plasma acid was stable, and it had small dependence on the discharge time. Therefore, by combining the time efficiency, the plasma discharge treatment of distilled water was determined. The best discharge time to obtain plasma acid was $600 \mathrm{~s}$, which was 10 minutes. The $\mathrm{pH}$ was 1.25 .

In our experiments, $1 \%$ and $1.5 \%$ chitosan solution were prepared by the plasma acid at $\mathrm{pH}$ of 1.25. The results showed that $1 \%$ chitosan could be dissolved in the plasma acid and a transparent

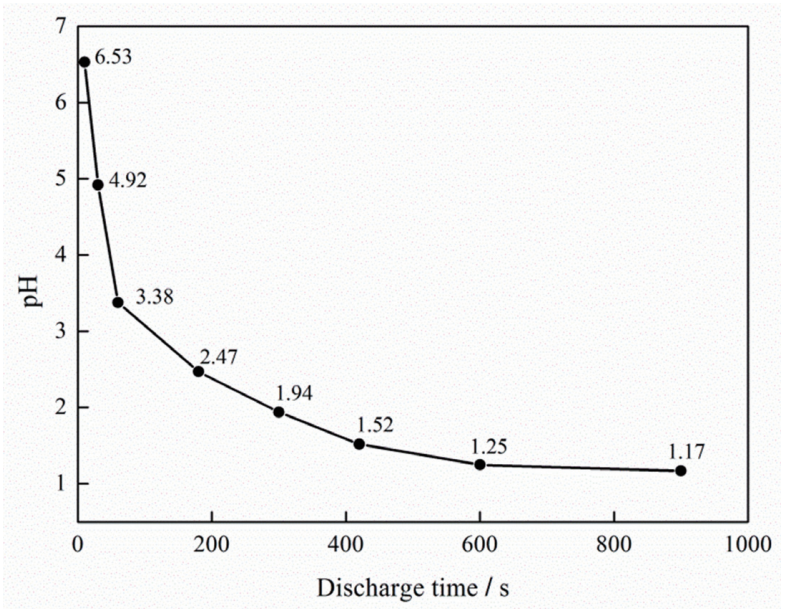

Fig. 1 The $\mathrm{pH}$ of plasma acid changed with discharge time solution could be obtained. However, $1.5 \%$ chitosan could not be dissolved completely, and some sediment at the bottom of bottle appeared. Then 1\% chitosan solutions were used in the following experiments. The electrical conductivity of $1 \%$ chitosan solution was $4.62 \mathrm{~ms} / \mathrm{cm}$, and the surface tension was $43.09 \mathrm{mN} / \mathrm{m}$.

Therefore, the conditions for the preparation of the membrane were as follows, $1 \%$ chitosan, the discharge power of $125 \mathrm{~W}$ and the discharge time of 10 minutes.

\subsection{Determination of timeliness of plasma acid}

The timeliness of plasma acid of $\mathrm{pH}=1.25$ was determined every $2 \mathrm{~h}$. The pH-Time relation curve was obtained and shown in Fig. 2. Seen from the chart, the initial $\mathrm{pH}$ value of plasma acid was 1.25. After standing for $4 \mathrm{~h}$, the $\mathrm{pH}$ became to 1.65 . The value of plasma acid was relatively stable. The $\mathrm{pH}$ change rate of per unit time $(/ \mathrm{h})$ was small. In the $4-8 \mathrm{~h}$ period, the acidity of plasma acid was unstable, changing from 1.65 to 4.06 . The change rate was larger. After $4 \mathrm{~h}$, it was shown that the acidity of the solvent varied greatly. In the $8-12 \mathrm{~h}$ period, the $\mathrm{pH}$ value was changed from 4.06 to 6.84 , and finally the plasma acid gradually became neutral.

In our experiment, the chitosan/plasma acid solution was prepared about 3-4 h, which was made in a relatively stable period of plasma acid. This result also illustrated that plasma acid is a green solvent, for it will become neutral after $12 \mathrm{~h}$. It needs no posttreatment of solvent.

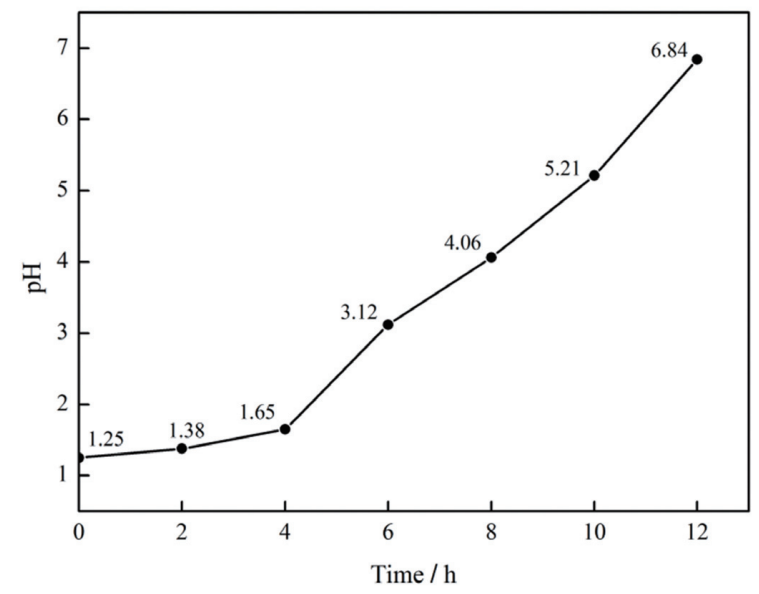

Fig. 2 The variation of $\mathrm{pH}$ value of plasma acid with time

\subsection{Properties of GO by XPS and TEM analysis}

As shown in Fig. 3, the TEM image of GO with different magnification was obtained at an accelerating voltage of $200 \mathrm{kV}$. As can be seen from the pictures, the GO has good dispersion, the surface 


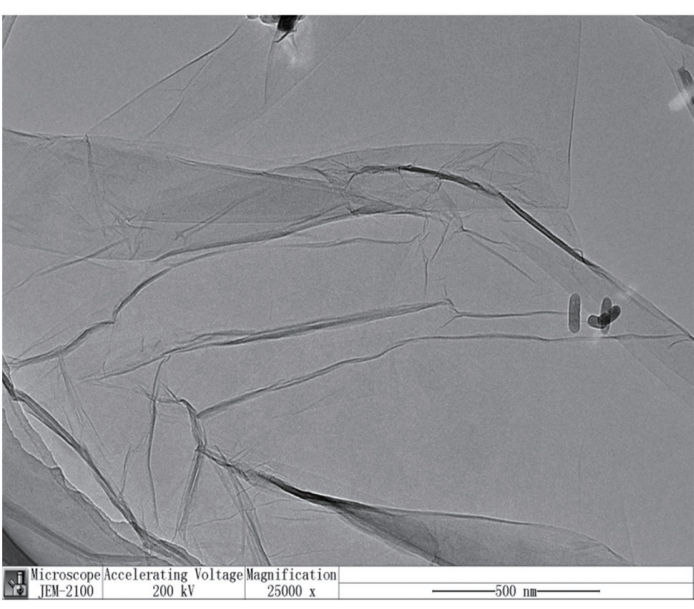

(a)

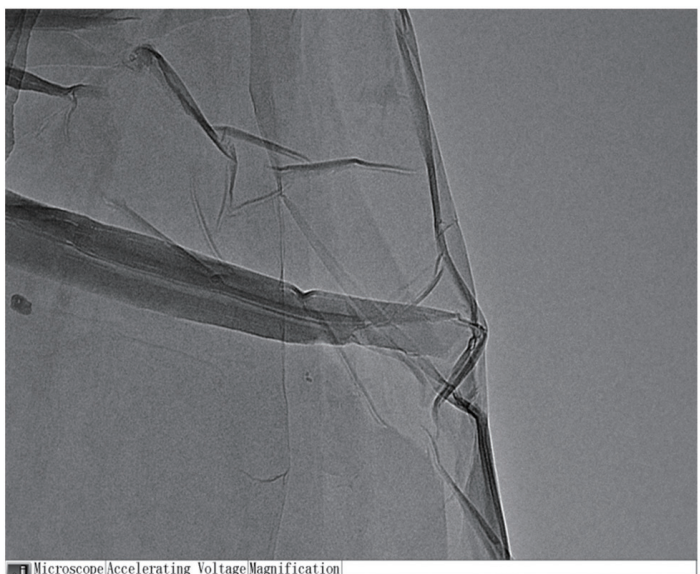

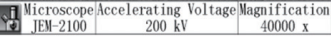

(b)

Fig. 3 The TEM pictures of GO

was smooth and transparent, and a large number of folds were appeared in the edge of the structure. It was shown that the GO membrane was very thin, at the same time, and it was also proved to have a monolayer molecular structure of GO. XPS spectrum

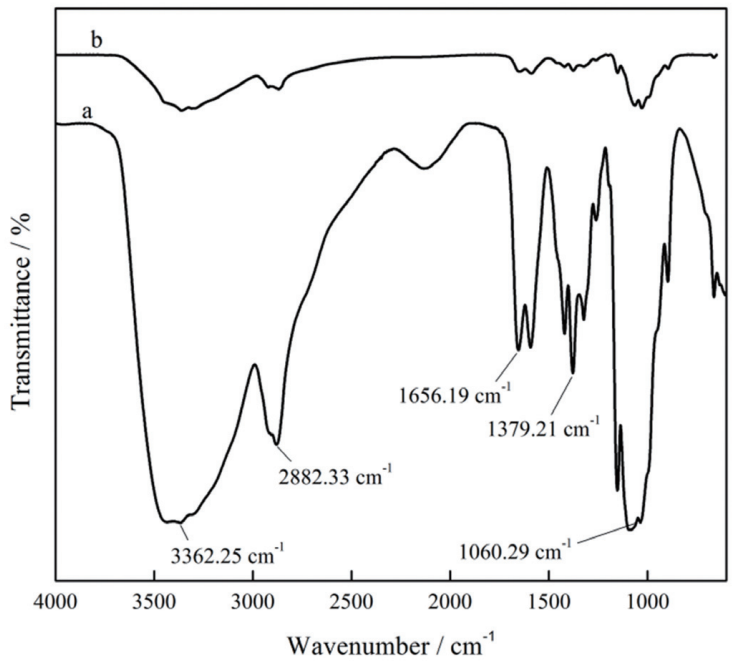

Fig. 4 FTIR of chitosan and GO/chitosan composite membrane (a) Chitosan, (b) GO/chitosan composite membrane scanning of GO showed that C $1 \mathrm{~s}$ was $68.2 \%$ and $\mathrm{O} 1 \mathrm{~s}$ was $31.7 \%$. The $\mathrm{O} / \mathrm{C}$ atomic ratio was calculated to be 0.46 .

\subsection{FTIR analysis of chitosan membrane}

Figure 4 was the FTIR of chitosan and GO/ chitosan composite membrane. For the FTIR curve of chitosan, the peak at $3362.25 \mathrm{~cm}^{-1}$ was the overlap band of - $\mathrm{OH}$ stretching vibration and - $\mathrm{NH}$ stretching vibration, the peak at $2882.33 \mathrm{~cm}^{-1}$ was the absorption band of $\mathrm{C}-\mathrm{H}$ stretching vibration, the peak at $1656.19 \mathrm{~cm}^{-1}$ was the absorption band of amide, the peak at $1379.21 \mathrm{~cm}^{-1}$ was the $-\mathrm{CH}_{3}$ symmetric vibration absorption band, and the peak at $1060.29 \mathrm{~cm}^{-1}$ was the vibrational absorption band of CC skeleton.

However, compared with chitosan membrane, there were no new peaks appeared in GO/chitosan composite membrane. The main component was chitosan after adding GO. Because of the low content of GO, the peaks which belongs of GO could not be seen in FTIR.

\subsection{XRD analysis of chitosan membrane}

Figure 5 was the XRD spectra of GO and GO/ chitosan composite membranes. From GO spectrum, there was a strong diffraction peak appearing at $2 \theta=$ 11.3 . For GO/chitosan composite membrane spectrum, two diffraction peaks of chitosan appeared at $2 \theta=20.62^{\circ}$ and $2 \theta=10.32^{\circ}$. They were the peaks of chitosan. But there was no characteristic diffraction peaks of GO. Therefore, from the disappearing of GO peaks in composite, it was indicated that the GO was uniformly distributed in the chitosan without agglomeration. The ordered structure of GO was formed in the chitosan matrix.

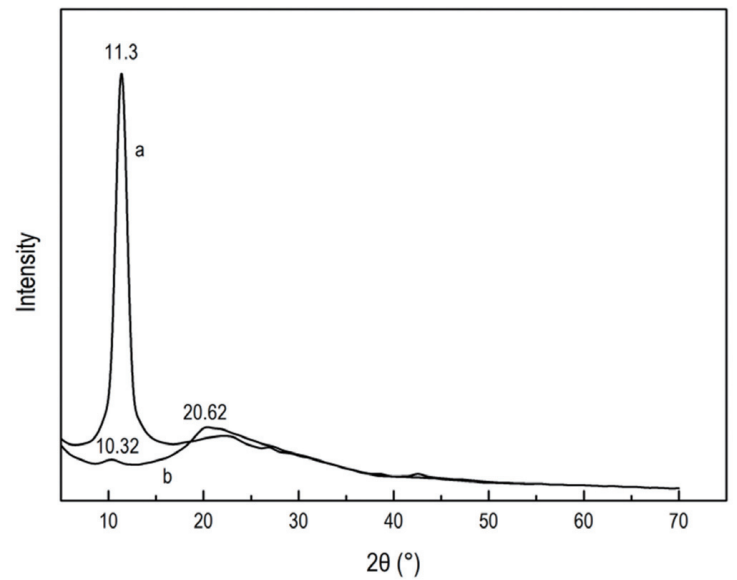

Fig. 5 XRD of GO and GO/chitosan composite membrane (a) GO, (b) GO/chitosan composite membrane 


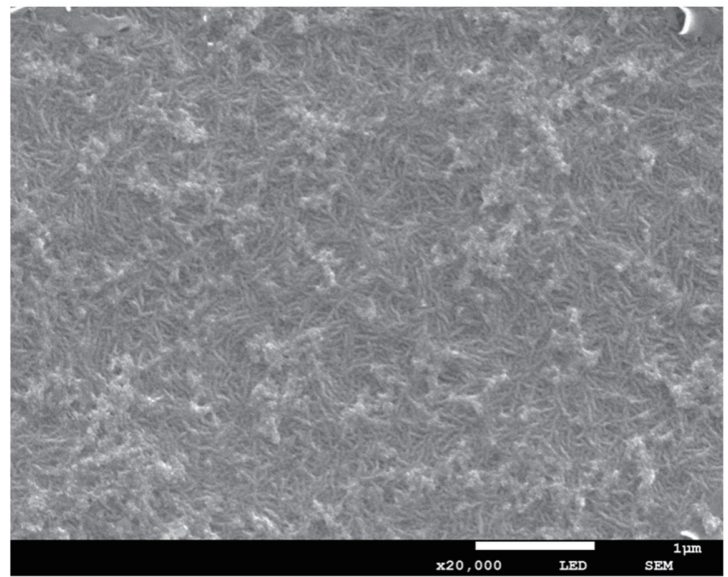

(a)

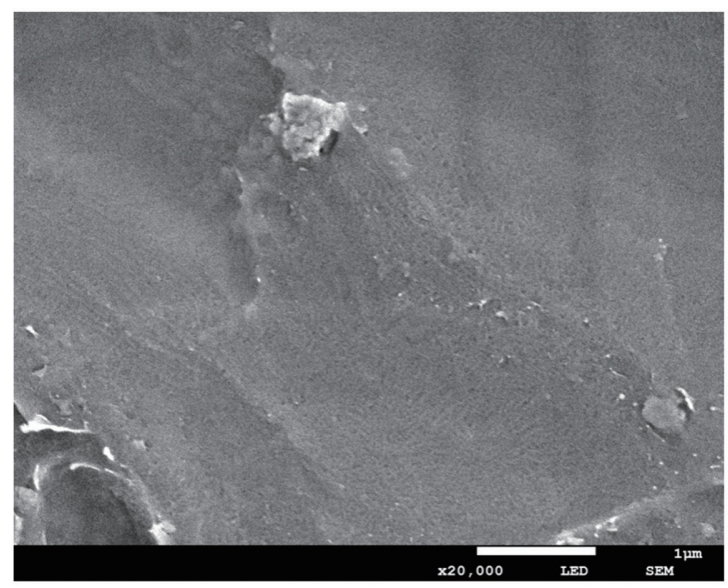

(b)

Fig. 6 SEM of chitosan membrane and GO/chitosan composite membrane (a) Chitosan membrane, (b) GO/chitosan composite membrane

\subsection{SEM analysis of chitosan membrane}

The surface morphology of chitosan membrane and GO/chitosan composite membrane were observed by the SEM and shown in Fig. 6. Without adding the GO, the surface of chitosan membrane had many strips. They were mainly caused by the polysaccharide chains of chitosan. After adding the $\mathrm{GO}$, the surface of $\mathrm{GO} /$ chitosan composite membrane became smooth. It was due to the well dispersion of GO in chitosan coming from the interaction between the hydroxyl groups in chitosan and the oxygen containing groups in GO. Therefore, the surface of GO/chitosan composite membrane was smooth compared with the chitosan membrane.

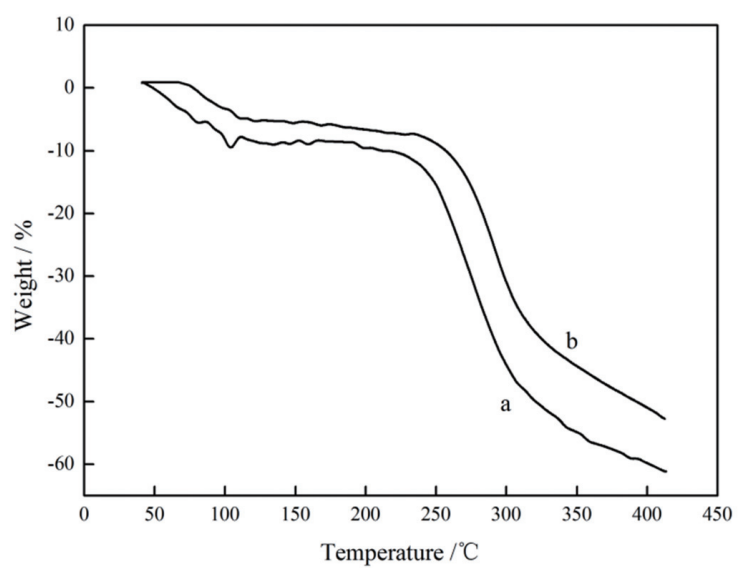

Fig. 7 TG of chitosan membrane and GO/chitosan composite membrane (a) Chitosan membrane, (b) GO/chitosan composite membrane

\subsection{TG analysis of chitosan membrane}

Figure 7 was the TG curves of chitosan membrane and GO/chitosan composite membrane. The addition of GO increased the thermal stability of the chitosan membrane. In the curve of chitosan membrane, the first weightlessness at $100^{\circ} \mathrm{C}$ was due to the evaporation of the water contained in the membrane. The second weightlessness at $250^{\circ} \mathrm{C}$ was due to the thermal degradation of a large number of chitosan molecules. However, for the GO/chitosan composite membrane, the second weightlessness appeared at $270^{\circ} \mathrm{C}$. This result illustrated that the thermal stability of chitosan membrane was increased after adding GO.

\subsection{Mechanical property analysis of chitosan membrane}

Chitosan membranes need to have a good mechanical strength. Its breaking strength and breaking elongation were shown in Table 2.

When the GO added, the strength of GO/chitosan composite membrane was enhanced from $0.61 \mathrm{MPa}$ to $1.48 \mathrm{MPa}$, increased by $143 \%$. But its elongation was decreased from $8.2 \%$ to $4.2 \%$. It may be due to the strong interfacial interaction between the homogeneously of dispersed GO and the chitosan matrix, which limited the movement and deformation of the molecular chain, thus increasing the strength of the composite membrane. However, the elongation

Table 2 Elongation and strength of membranes

\begin{tabular}{ccc}
\hline Membranes & Elongation (\%) & Strength (MPa) \\
\hline Chitosan membrane & $8.2 \pm 0.2$ & $0.61 \pm 0.03$ \\
GO/chitosan composite membrane & $4.2 \pm 0.3$ & $1.48 \pm 0.12$ \\
\hline
\end{tabular}


was decreased from $8.2 \%$ to $4.2 \%$, the material exhibited brittle, possibly due to the interfacial interaction was too strong between GO and chitosan matrix. At present, the brittleness mechanism of this kind of composite material was not very clear, so it was necessary to study further.

\section{Conclusions}

In this paper, plasma acid was used as a green solvent to prepare chitosan membrane and GO/ chitosan composite membrane. After adding GO, the properties of the membrane were changed. FTIR showed that there were no new peaks appeared in $\mathrm{GO} /$ chitosan composite membrane, its main component was still chitosan. XRD showed that the diffraction peaks of chitosan was appeared at $2 \theta=$ $20.62^{\circ}$ and $2 \theta=10.32^{\circ}$, without characteristic diffraction peaks of GO, it was illustrated that GO dispersed uniformly in the chitosan matrix. Due to the interaction between the hydroxyl groups in chitosan and the oxidation groups in GO, the surface of GO/ chitosan composite membrane became smooth and the thermal stability of $\mathrm{GO} /$ chitosan composite membrane was increased. Besides, it also had good mechanical properties. Therefore, plasma acid was used as a green and environmentally-friendly solvent, it would have extensive applications.

\section{Acknowledgements}

This study was supported by Liaoning Province Colleges' Outstanding Talent Support Program [grant number LJQ 2015008] and Liaoning Province Natural Science Foundation [grant number 20170540064],
China.

\section{References}

1. W. Zhang, H. Lin and Y. Y. Chen. Journal of Nantong University (Natural Science Edition), 5, 30 (2006).

2. Y. Q. Zhao and Y. Fang. Guizhou Chemical Industry, 26, 11 (2001).

3. Y. G. Wu, D. Ye, SH.Y. Qiu, et al. Journal of Guizhou Polytechnic University of Technology (Natural Science Edition), 34, 52 (2005).

4. C. J. Liu and J. J. Zou. Journal of Tianjin University, 37, 189 (2004).

5. ZH. P. Yin. China Elastomerics. 13, 52 (2003).

6. SH. He, J. M. Liang, H. L. Hu, et al. Materials Review B: Research Papers, 30, 1 (2016).

7. S. Gilje, S. Han, M. SH. Wang, et al. Nano Lett., 7, 3394 (2007).

8. SH. P. Fu, X. J. Wan, Y. SH. Chen, et al. Small, 5, 1682 (2009).

9. Z. W. Ma and H. C. Ge. Material report, 28, 61 (2014).

10. J. Zhang, Q. M. Ye. Silicone Mater (China), 23, 31 (2009).

11. Y. Yang, H. Kim, A. Starikovskiy, Y. Cho A. Fridman. Plasma Chemistry and Plasma Processing, 31, 51 (2011).

12. P Baroch, N Satio, O Takai. J. Phys. D: Appl Phys, 41, 085207 (2008).

13. M. Yusupov, E. C. Neyts, P. Simon, G. Berdiyorov, R. Snoeckx, AC. T. van Duin, A. Bogaerts. J. Phys. D: Appl Phys, 47, 025205 (2014).

14. CH. J. Liu and J. J. Zou. Journal of Tianjin University, 37, 189 (2004). 